\title{
HISTOIRE ET THÉORIE DU HASARD À L'ÂGE CLASSIQUE SELON COURNOT
}

\author{
Thierry MARTIN
}

\begin{abstract}
RÉsumé: Dans un article décisif sur la naissance du calcul des probabilités (1970), Ernest Coumet rappelait la formule de Cournot selon laquelle le retard qui marque la naissance de la théorie du hasard est « un pur effet du hasard ». On se propose ici de soumettre ce jugement à l'examen critique non de l'histoire, mais de la pensée même de Cournot; sa philosophie de l'histoire et sa représentation de l'histoire des sciences au XVII ${ }^{\mathrm{e}}$ siècle semblant, tout d'abord, dénoncer par avance un jugement aussi péremptoire. Pour rendre compte de ce qui peut alors apparaître de sa part comme une inconséquence, voire un aveuglement, on forme l'hypothèse que cette affirmation constitue un artefact de sa conception internaliste de l'histoire des sciences et des révolutions scientifiques. En ce sens, l'article constitue une illustration de la nécessité théorique, mais aussi des difficultés de l'histoire des mathématiques.
\end{abstract}

Mots-CLÉs : Cournot, probabilités, histoire des sciences, hasard, révolution scientifique.

ABSTRACT: In a decisive article about the birth of the calculus of probabilities (1970), Ernest Coumet recalled Cournot's formula: the delay concerning the birth of the theory of chance is " a pure effect of chance ». We suggest here submitting this judgement to the critical examination not of history but of Cournot's very thought; his philosophy of history and his representation of the history of science in the XVII century seems, at first, to denounce beforehand such a decisive judgement. To render an account of what can then appear on his part to be an inconsistency or even a blindness, we formulate the hypothesis that this statement constitutes an artefact of his internal conception of the history of science and scientific revolutions. In that sense, the article constitutes an illustration of theoretical necessity and of the difficulties of mathematical history as well.

KEYWORDS : Cournot, probabilities, history of science, chance, scientific revolution.

Revue de synthèse : $4^{\mathrm{e}}$ sér., ${ }^{\mathrm{os}}$ 2-3-4, avr.-déc. 2001, p. 455-471. 
Zusammenfassung: In einem entscheidenden Artikel über die Entstehung der Wahrscheinlichkeitsrechnung (1970) erinnerte Ernest Coumet an den Satz von Cournot, nach welchem die verspätete Entstehung der Wahrscheinlichkeitsrechnung «der reine Effekt des Zufalls » ist. Hier nehmen wir uns vor, diese Feststellung der kritischen Prüfung nicht etwa der Geschichte sondern des Cournotschen Denkens selbst zu unterwerfen. Cournots Philosophie der Geschichte und seine Vorstellung der Wissenschaftsgeschichte im 17. Jahrhundert scheinen zuerst solch ein endgültiges Urteil im voraus anzuprangern. Um das zu begreifen, was uns dann von seiten Cournots als Inkonsequenz - ja als Verblendung - erscheinen mag, stellen wir hier die Hypothese auf, diese Behauptung bilde ein Artefakt des Cournotschen internalistischen Begriffs der Wissenschaftsgeschichte und der wissenschaftlichen Revolutionen. In diesem Sinne stellt der Artikel eine Illustration der theoretischen Notwendigkeit sowie der Schwierigkeiten der Mathematikgeschichte dar.

STICHWÖRTER: Cournot, Wahrscheinlichkeiten, Wissenschaftsgeschichte, Zufall, wissenschaftliche Revolution.

Thierry Martin, né en 1950, est maître de conférences en philosophie des sciences à l'université de Besançon et chercheur au laboratoire de Recherches philosophiques sur les logiques de l'agir (CNRS EA 2274-GDR 2226). Ses recherches concernent principalement l'histoire et la philosophie des probabilités.

Adresse: Université de Franche-Comté, 30 rue Mégevand, F-25030 Besançon Cedex.

Courrier électronique : thierry.martin@univ-fcomte.fr 
Dans un article décisif ${ }^{1}$ sur les conditions d'émergence du calcul des probabilités, «La théorie du hasard est-elle née par hasard ? », Ernest Coumet rappelait la formule d'Antoine-Augustin Cournot selon laquelle le retard qui marque la naissance de la théorie du hasard est « un pur effet du hasard », Cournot allant même jusqu'à prétendre que « rien ne s'opposait à ce qu'un Grec de Cos ou d'Alexandrie eût pour les spéculations sur les chances le même goût que pour les spéculations sur les sections du cône ${ }^{2}{ }$.

Certes, Cournot ne fait preuve d'aucune originalité en relevant le caractère anecdotique des origines du calcul des probabilités ${ }^{3}$, mais une telle formule sous sa plume est cependant étonnante. Déjà, comme le remarque Coumet, elle tranche avec le style d'ordinaire si prudent de Cournot. Mais, plus gravement, c'est avec le contenu de sa pensée qu'elle semble s'opposer, et cela pour trois raisons au moins :

$1^{\circ}$ On peut, tout d'abord, remarquer qu'elle s'accorde mal avec l'orientation qui dirige sa philosophie de l'histoire. Celle-ci, définie comme « étiologie historique », se donne en effet pour objet «l'analyse et la discussion des causes ou des enchaînements de causes qui ont concouru à amener les événements dont l'histoire offre le tableau; causes qu'il s'agit surtout d'étudier, précise-t-il, au point de vue de leur indépendance ou de leur solidarité ${ }^{4} \gg$. La critique historique vise ainsi à identifier, dans le mouvement historique, la part respective du fortuit et du nécessaire, de l'essentiel et de l'accidentel, pour dégager, sous le détail des événements et dans la multiplicité des facteurs qui les engendrent, les causes profondes qui en rendent raison. Or, concernant l'émergence du calcul des probabilités, force est de reconnaître qu'il n'y a pas, de la part de Cournot, discussion des causes, pas même analyse. Il pose comme allant de soi que « le hasard historique tient une très grande place dans l'histoire de la théorie du hasard ${ }^{5} »$. C'est alors l'intelligibilité de cette émergence qui se trouve compromise, puisque du hasard on ne peut rendre raison.

1. Coumet, 1970.

2. Counnot, $1875, \mathrm{IV}^{\mathrm{e}}$ sect., $\S 4 ; 1979$, p. 180 (dans la suite, les numéros de page renvoient, comme ici, à l'édition des CEuvres complètes citée comme OC, voir Cournot, 1973-).

3. Déjà, comme le rappelle Coumet, 1970, Leibniz notait que le calcul des probabilités naquit à « l'occasion » de la rencontre de Pascal et du chevalier de Méré, voir LeibNIz, 1886, liv. IV, chap. XVI. Gouraud, 1848, p. 3, écrit de même en 1848 que «l'occasion la plus imprévue peut susciter soudain la découverte la plus rare ». Et Du PASQuIER, 1926, p. 5, reprenant Cournot, affirmera de la théorie du hasard que «le hasard voulut qu'elle fût elle-même due à un hasard ».

4. Cournot, 1872, liv. I, chap. I; 1973, p. 10.

5. Cournot, 1872, liv. III, chap. I; 1973, p. 184. 
$2^{\circ}$ La formule ne s'accorde pas davantage avec sa représentation de l'histoire des sciences au XVII ${ }^{\mathrm{e}}$ siècle, laquelle, selon Cournot, manifeste au plus haut point «l'irrésistible nécessité des causes générales qui ne pouvaient manquer d'amener, un peu plus tôt, un peu plus tard, le triomphe de l'idée ${ }^{6}$ que des savants exceptionnels avaient su apercevoir. S'il est vrai qu'au XVII ${ }^{\mathrm{e}}$ siècle «le tissu de l'histoire scientifique est déjà si serré, les grandes découvertes s'y pressent tellement, qu'on sent bien qu'elles étaient mûres ${ }^{7} »$, comment comprendre l'affirmation de la fortuité de l'acte de naissance du calcul des probabilités? Doit-on reconnaître que Cournot se soit laissé aller ici à une incohérence, ou bien faut-il admettre que le calcul des probabilités constitue une exception, et une exception singulière, dans l'ensemble du mouvement scientifique de l'âge classique? Dans l'un ou l'autre cas, la situation du calcul des probabilités fait difficulté et exige d'être éclaircie.

$3^{\circ}$ Enfin, peut-on sérieusement affirmer qu'un penseur grec de l'Antiquité pouvait se proposer de mathématiser notre conduite face à l'incertain? Si le XVII ${ }^{\mathrm{e}}$ siècle scientifique est profondément révolutionnaire, c'est parce qu'aux dires de Cournot lui-même ${ }^{8}$, il conduit à substituer à une attitude essentiellement contemplative marquant la pratique des «sciences abstraites » antiques, la volonté de leur faire jouer un rôle déterminant dans la connaissance du monde réel, celui de fournir la «clef» de son intelligibilité. En conséquence, Cournot historien $\mathrm{du} \mathrm{XVII}^{\mathrm{e}}$ siècle rétorquerait à Cournot historien du calcul des probabilités que «les spéculations sur les chances » ne mobilisent pas le même intérêt théorique que celles portant sur les sections coniques et n'engagent pas la même représentation de la nature et du rôle des mathématiques.

Comment alors comprendre la présence d'un tel jugement dans l'œuvre de Cournot? On pourrait certes avancer qu'il se laisse emporter par le plaisir de faire un bon mot. C'est sans doute en partie vrai, mais l'explication est insuffisante, d'autant que Cournot récidive, cette invocation du hasard à l'origine du calcul des probabilités intervenant à deux reprises, d'abord dans les Considérations, ensuite dans Matérialisme, vitalisme, rationalisme. Cette remarque permet d'exclure également l'hypothèse d'une erreur de jeunesse, puisque, justement, ces deux ouvrages sont parmi les derniers publiés par Cournot.

En conséquence, les trois raisons précédentes conservent leur force, et leur confrontation avec la formule lapidaire en appelant au hasard dans la

6. Cournot, 1872, liv. III, chap. I; 1973, p. 174-175.

7. Cournot, 1872, liv. III, chap. I; 1973, p. 174.

8. Cournot, 1872, liv. III, chap. I; 1973, p. 173-174. 
naissance du calcul des probabilités compromet, semble-t-il, la cohérence de la pensée de Cournot.

Afin d'éclairer cette triple difficulté, il convient déjà de restituer le sens de cette formule, et pour cela de mieux préciser le mode d'intervention du hasard dans l'histoire. Le hasard, pour Cournot, ne se réduit ni au surgissement imprévu et inconditionné d'un effet sans cause, ni à une appréhension subjective résultant de notre ignorance des causes de l'événement; il désigne la rencontre accidentelle entre plusieurs séries de faits ou de causes indépendants. Ce qui fait la fortuité n'est donc pas l'absence de détermination, ou son ignorance, mais l'absence de nécessité dans la conjonction des déterminations. En ce sens, un événement peut être le produit d'un ensemble de causes qui le déterminent, tout en étant fortuit dès lors qu'il n'est pas nécessaire. Par conséquent, invoquer le hasard à propos de la naissance du calcul des probabilités, ce n'est pas verser dans l'irrationnel ou en appeler au mystère d'une inspiration qui aurait visité Blaise Pascal et Pierre Fermat, c'est, pour Cournot, signifier que dans la nature de cette discipline rien n'exigeait qu'elle ait dû apparaître au $\mathrm{XVII}^{\mathrm{e}}$ siècle et grâce aux travaux de Pascal et de Fermat précisément. Pour parler le langage de Cournot, il faut dire qu'il y a certes des causes à cet événement, mais il n'y a pas de raison pour qu'il intervienne à ce moment précis de l'histoire des sciences.

Cette distinction entre cause et raison est au cœur de la représentation cournotienne de l'histoire. En effet, le but que Cournot assigne à l'étiologie historique est moins de mettre au jour les causes des faits historiques que de les ordonner, de les hiérarchiser pour discerner parmi ces causes celles qui sont déterminantes. Il s'agit de démêler dans le spectacle de l'histoire, sous l'exubérance des événements plus ou moins fortuits et accidentels, les grandes lignes de force, les tendances générales qui en permettent l'intelligibilité. Et ce partage exige que l'on distingue entre les causes immédiates et souvent accidentelles, responsables de ce que l'événement a de singulier, des conditions déterminantes qui rendent raison de ses propriétés, et par là, en permettent l'intelligibilité.

Cette distinction, héritée de l'analyse statistique, trouve à s'appliquer, dans le champ des sciences de la nature ou des sciences sociales, à des séries d'épreuves indépendantes, permettant alors de dégager des constantes, donc de faire apparaître la raison du phénomène derrière les causes accidentelles et irrégulières. En histoire, où l'on n'a plus affaire à des séries d'événements indépendants, elle aide à discerner des permanences, des conditions déterminantes et relativement stables qui rendent raison du fait historique derrière les causes immédiates engendrant l'événement : 
«Si l'on tient à une parfaite exactitude de langage, il faudra dire que l'étiologie ou la philosophie de l'histoire s'enquiert de la raison des événements plutôt que de la cause des événements. Car, l'idée de cause implique celle d'une action, d'une force douée de son énergie propre; et ce que la critique historique doit mettre en évidence, ce sont le plus souvent des résistances passives, des conditions de structure et de forme qui prévalent à la longue et dans l'ensemble des événements sur les causes proprement dites, sur celles qui interviennent avec le mode d'activité qui leur est propre, dans la production de chaque événement en particulier. Si l'on projette un dé un grand nombre de fois, et que l'as reparaisse beaucoup plus souvent que les autres points, ce sera l'indice de quelque irrégularité de structure ou de distribution de la masse, qui seule peut rendre raison de la fréquence observée, mais qui n'intervient que passivement, par suite de l'inertie de la matière du dé, et qui dès lors n'est point une cause, selon la rigueur du terme ${ }^{9}$. »

Cependant, si la philosophie de l'histoire de Cournot se détourne ainsi d'une histoire événementielle pour considérer les facteurs déterminants qui rendent raison des événements, ces «conditions de structure et de forme qui prévalent à la longue » ne sont ni les conjonctures économiques et sociales, ni les données géographiques ou climatiques de la longue durée que mettra en avant l'École des Annales, mais désignent des configurations culturelles qui commandent ce qu'il appelle la «tendance générale ${ }^{10}$ » propre à une époque historique donnée, ou encore les « faits généraux » qui impriment à une époque ce que Cournot appelle son «allure générale » :

«La philosophie de l'histoire a essentiellement pour objet de discerner dans l'ensemble des événements historiques des faits généraux, dominants, qui en forment comme la charpente ou l'ossature; de montrer comment à ces faits généraux et de premier ordre s'en subordonnent d'autres, et ainsi de suite jusqu'aux faits de détail qui peuvent encore offrir un intérêt dramatique, piquer vivement notre curiosité, mais non notre curiosité de philosophes ${ }^{11}$. »

Il faut donc distinguer les faits historiques selon leur degré de généralité, et les hiérarchiser en fonction de leur pouvoir déterminant et de leur rythme différencié, afin de mettre à jour «l'allure générale », soit les traits dominants, d'une époque historique.

Si la date de naissance du calcul des probabilités est alors un fait de hasard, c'est que son émergence est indépendante de cette allure générale, de cette configuration spirituelle propre au $\mathrm{XVII}^{\mathrm{e}}$ siècle. Et la question se pose alors de savoir d'une part quelle est cette allure générale, et de l'autre en quoi le calcul des probabilités lui est étranger.

9. Cournot, 1872, liv. I, chap. i; 1973, p. 15-16 (souligné par l'auteur).

10. Cournot, 1872, liv. I, chap. I; 1973, p. 13.

11. Cournot, $1861, \S 546 ; 1982$, p. 489. 
Le XVII ${ }^{\mathrm{e}}$ siècle se caractérise aux yeux de Cournot par deux traits principaux :

$1^{\circ}$ Tout d'abord, il est le siècle qui voit s'accomplir les révolutions scientifiques fondatrices de la science moderne:

«Ce sont les progrès et les révolutions des sciences au XviI ${ }^{e}$ siècle qui lui impriment ce caractère singulier et exceptionnel de grandeur, que ni la religion, ni la politique, ni la philosophie, ni les lettres, ni les arts ne lui communiqueraient à ce degré éminent ${ }^{12}$. »

Le développement des connaissances scientifiques s'effectue, indique Cournot ${ }^{13}$, par un double mouvement : le perfectionnement de la science, fruit d'une élaboration intellectuelle par laquelle la science améliore son organisation interne, l'accroissement des connaissances, qui trouve, lui, sa source dans le rapport au réel, résultant d'observations nouvelles et de la mise au jour de faits demeurés inaperçus. Ces nouveaux matériaux exigeant d'être intégrés à la connaissance peuvent entrer en conflit avec les principes d'ordre admis jusque-là et faire apparaître de nouveaux rapports entre les phénomènes, appelant alors une réorganisation principielle de la science. C'est ce divorce entre les principes explicatifs en vigueur et les nouvelles observations qui, selon Cournot, engendre les révolutions scientifiques. Mais, pour que celles-ci s'accomplissent, deux conditions sont encore nécessaires : premièrement, il faut que la science ait atteint une maturité suffisante pour pouvoir assimiler les enseignements à tirer du désaccord entre les faits expliqués et les principes jusqu'ici adoptés; secondement, et surtout, il faut que naisse une «idée nouvelle », ou encore une «idée heureuse », permettant de profiter de cette maturité, c'est-à-dire d'engendrer une théorie explicative.

Cette conjonction entre la maturité de la science et une individualité ou des individualités - capable(s) de la mettre à profit, si elle est la condition de toute découverte scientifique, est particulièrement déterminante s'agissant d'une révolution scientifique, où il est nécessaire que la communauté scientifique compte parmi ses membres des individualités exceptionnelles, aptes, justement, à mener à bien cette entreprise de réorganisation du discours scientifique.

C'est ici que peut se révéler, dans l'histoire des sciences, l'une des formes d'interventions du hasard: dans la rencontre partiellement fortuite

12. Cournot, 1872, liv. III, chap. I; 1973, p. 172.

13. Cournot, $1851, \S 308 ; 1975$, p. 363-364. 
d'une maturité de la science qui, à la fois, rend possible et exige un développement scientifique, et d'une individualité — ou de plusieurs individualités - susceptible(s) de profiter de cette maturité.

Cette part de hasard peut être mineure, ce qui a lieu lorsque la science a atteint un tel degré de maturité qu'elle n'intervient que pour hâter ou retarder légèrement cette réorganisation. Et c'est, comme on l'a vu, l'un des traits des grandes découvertes du $\mathrm{XVII}^{\mathrm{e}}$ siècle, puisqu'elles pouvaient arriver, dit Cournot, «un peu plus tôt, un peu plus tard », mais ne pouvaient pas ne pas advenir.

À ce titre, le XVII ${ }^{\mathrm{e}}$ siècle occupe une place privilégiée dans l'histoire des sciences, non seulement par la richesse de sa production, mais également parce qu'il réalise au plus haut point les caractères de l'historicité, c'est-àdire cette combinaison de nécessité interne et de hasard, de raison déterminante et de causes accidentelles qui est, selon Cournot, la marque de l'histoire :

«Antérieurement au xvII ${ }^{\mathrm{e}}$ siècle, les progrès des sciences sont si lents, les découvertes notables si clairsemées, que le tableau qu'on en peut tracer ressemble plus à des fastes, à des registres ou à des annales monacales ou hiératiques, qu'à une composition vraiment historique, où l'intérêt ressort de la forte et intime union de toutes les parties du récit, autant que de la puissante action des personnages mis en scène. Plus tard, et lorsqu'une légion de travailleurs, dont chacun vise à l'estime de ses pairs et à une réputation viagère bien plus qu'à la gloire, fouilleront en tous sens ce sol où leurs illustres devanciers ont ouvert les routes magistrales, la revue, le compte rendu, c'est-à-dire le journal et la gazette remplaceront l'histoire, comme l'histoire avait remplacé les sèches et courtes mentions des temps plus anciens ${ }^{14}$. »

Si le hasard joue un rôle limité dans le développement scientifique dès lors qu'il y a quasi simultanéité entre l'état de maturité de la science et la présence d'une individualité géniale apte à profiter de cette maturité, on peut en déduire qu'inversement l'intervention du hasard sera plus nette lorsqu'il y aura décalage entre l'état de maturité de la science et le moment de la découverte ou de l'invention. Et c'est bien ce décalage que note Cournot en écrivant que l'Antiquité grecque avait les moyens d'ébaucher une mathématisation du hasard. La science mathématique avait atteint une maturité suffisante à l'émergence de ce nouveau champ bien avant les travaux de Pascal et de Fermat.

L'affirmation selon laquelle la date de naissance du calcul des probabilités est un fait de hasard transcrit donc ce constat selon lequel la construction de ses premières règles ne nécessitait pas la mise au point d'instru-

14. Cournot, 1872, liv. III, chap. I; 1973, p. 174. 
ments mathématiques différents de ceux que possédait déjà l'Antiquité. Tel est bien le sens de la remarque de Cournot, puisque, précise-t-il,

« les solutions qui ont piqué la curiosité de Pascal et de Fermat n'étaient pas de celles qui ne peuvent (comme l'invention du calcul infinitésimal et de la mécanique rationnelle) venir qu'à la suite d'une longue élaboration scientifique. L'esprit subtil des Grecs était capable de les trouver comme il en a trouvé de plus difficiles ${ }^{15} »$.

À quoi il ajoutera, trois ans plus tard, que l'analyse mathématique des jeux de hasard, point de départ du calcul des chances, n'exigeait que «quelques notions très simples d'arithmétique ou de géométrie ${ }^{16}$ ».

Il demeure cependant une difficulté. Invoquer le hasard pour rendre compte du décalage temporel entre les possibilités techniques d'émergence d'un savoir et sa date réelle de naissance ne permet en rien de rendre compte de ce décalage; et l'argument est particulièrement insuffisant quand cet avènement constitue une révolution scientifique. Or tel est bien le cas du calcul des probabilités, puisque le premier chapitre du livre III des Considérations se proposant d'étudier « la révolution des mathématiques » au XVII ${ }^{\mathrm{e}}$ siècle considère deux domaines : le calcul infinitésimal et le calcul des probabilités.

Comment comprendre alors que cette révolution des mathématiques, possible depuis l'Antiquité, ne se réalise effectivement que vingt siècles plus tard? Le second trait caractéristique du XVII ${ }^{\mathrm{e}}$ siècle peut aider à éclairer ce point.

$2^{\circ}$ Il ne suffit pas que la réorganisation principielle de la science lui permette d'intégrer de nouveaux faits pour que l'on assiste à une révolution scientifique, car cette restructuration pourrait n'être qu'artificielle et passagère. Il faut encore que, par là, la science se donne les moyens de rendre compte des rapports réels qui régissent les phénomènes. Le mérite de Galilée tient justement au changement de méthode opéré par lui et grâce auquel la raison possède le pouvoir non seulement de se rendre intelligible le réel, mais encore de mesurer et de vérifier cet accord entre l'explication rationnelle et l'ordre des phénomènes :

« De tout temps le genre humain avait senti le besoin de l'observation et de l'expérience, avait vécu d'observations bien ou mal conduites, rattachées tant bien que mal à des théories plus ou moins aventureuses : mais l'expérience précise, numérique, quantitative, et surtout l'expérience indirecte qui utilise les

15. Cournot, 1872, liv. III, chap. I; 1973, p. 184.

16. Cournot, 1875, IV $^{\mathrm{e}}$ sect., $\S 4 ; 1979$, p. 180. 
relations mathématiques pour mesurer, à l'aide de grandeurs sur lesquelles nos sens et nos instruments ont prise, d'autres grandeurs insaisissables directement, à cause de leur extrême grandeur ou de leur extrême petitesse, voilà ce dont les plus doctes n'avaient pas l'idée ${ }^{17}$. »

Or, c'est justement cette réorientation des mathématiques en direction de leur pouvoir d'informer le réel qui forme le second trait caractéristique du $\mathrm{XVII}^{\mathrm{e}}$ siècle aux yeux de Cournot. Le XVII ${ }^{\mathrm{e}}$ siècle est en effet ce moment où la raison ne se limite plus à une visée purement spéculative, mais se voit reconnaître le pouvoir de rendre compte du réel :

«L'histoire des sciences au XVII ${ }^{\mathrm{e}}$ siècle marque justement cette époque où les sciences abstraites, longtemps cultivées pour elles-mêmes et pour le charme que quelques esprits y trouvent, ou par un secret et vague pressentiment de leur rôle futur, donnent tout à coup la clef de ce qu'il y a de plus fondamental, de plus simple [...] dans l'ordre de l'univers [réalisant par là] la merveilleuse alliance des spéculations abstraites et des observations judicieusement discutées ${ }^{18}$.»

Sans doute, cette maîtrise rationnelle du réel s'illustre de manière privilégiée dans la mise au point de la mécanique. Mais, ajoute Cournot,

«si la mécanique rationnelle est l'une des grandes voies par où les mathématiques nous font pénétrer dans l'économie du monde, il y en a une autre dont la théorie des combinaisons donne la clef, voie plus raboteuse, moins imposante, moins large à la première vue, quoique ouvrant des accès dans des directions bien plus variées, et dont la découverte, sinon l'aplanissement, appartient aussi au XVII ${ }^{\mathrm{e}}$ siècle $^{19} »$.

La naissance du calcul des probabilités se trouve ainsi placée dans le prolongement de ce mouvement de mathématisation du réel, caractéristique du XVII ${ }^{e}$ siècle. Mais, du même coup, les affirmations initiales soulignant la fortuité de l'acte de naissance du calcul des probabilités se trouvent sinon réfutées, du moins malmenées.

D'une part, il apparaît que la date de naissance du calcul des probabilités n'est pas à mettre au compte du seul hasard, mais résulte en grande partie de cette mutation du statut de la connaissance, faisant de la science l'instrument d'une maîtrise mathématisée du réel.

D'autre part, c'est l'affirmation selon laquelle la mathématique grecque aurait pu s'atteler à une mathématisation du hasard qui se trouve mise en

17. Cournot, 1872, liv. III, chap. II; 1973, p. 187.

18. Cournot, 1872, liv. III, chap. I; 1973, p. 173-174.

19. Counnot, 1872, liv. III, chap. I; 1973, p. 182. Voir, également, Cournot, 1851, § 346; 1975 , p. 413. 
cause. Elle l'est davantage encore, lorsqu'on la rapporte à d'autres textes où Cournot met justement l'accent sur le caractère contemplatif de la mathématique grecque. Ainsi, il écrit au début des Considérations :

«Au fond, l'utilité pratique, du genre de celle d'une arithmétique élémentaire ou usuelle, n'était pas ce dont les Grecs se souciaient beaucoup : ils tenaient bien plus à la rigueur, même pointilleuse, de la démonstration, à l'élégance idéale d'une construction dont un peu de sable faisait tous les frais matériels, et qui presque jamais n'était destiné à devenir ce que nous appelons une épure ${ }^{20}$.»

Il faut donc reconnaitre qu'il existe une sorte de divorce entre la formule lapidaire de Cournot invoquant le rôle du hasard dans la naissance du calcul des probabilités et l'orientation de son analyse du développement scientifique au XVII siècle.

Tout se passe comme si Cournot ne s'apercevait pas que son analyse lui fournissait des éléments permettant d'aller au-delà du simple constat de l'intervention d'un hasard historique. Comment alors comprendre ce qui s'offre comme un aveuglement de Cournot à l'égard des conséquences de sa propre pensée?

D'une certaine façon, on peut dire qu'il n'y a pas ici d'aveuglement de Cournot, car si, effectivement, le hasard n'est pas absence de cause, mais absence de nécessité, dire qu'il joue un rôle dans la naissance du calcul des probabilités, c'est tout simplement signifier que cette naissance n'était pas nécessaire, qu'elle existe de fait, donc est une donnée historique.

Cependant l'ambiguïté demeure, car placer la naissance du calcul des probabilités dans le prolongement de l'effort de mathématisation du réel ou insister sur le décalage temporel entre la naissance effective d'un calcul et sa possibilité technique d'apparition ne revient pas au même.

Dans la mesure où l'affirmation de la fortuité de l'acte de naissance du calcul des probabilités prend appui sur le constat de la forme techniquement élémentaire des instruments mathématiques mobilisés par les premiers probabilistes, on peut alors supposer que s'expriment dans la formule de Cournot, non seulement l'ignorance des interdits théologicojuridiques qui pesaient initialement sur les pratiques aléatoires — interdits qu'étudie Coumet dans l'article cité plus haut —, mais aussi un point de vue internaliste par lequel sont seulement prises en compte les conditions techniques du procès de mathématisation au détriment des conditions culturelles de la maitrise rationnelle du hasard. Or, justement ici, un tel point de vue est insuffisant, la géométrie du hasard se donnant initialement un objet qui n'est pas spécifiquement mathématique.

20. Cournot, 1872, liv. I, chap. III, p. 33. 
Pour rendre compte de la date de naissance de la mathématisation du hasard, il fallait, comme l'a montré Coumet, prendre en considération les conditions culturelles qui la rendent possible, à savoir la neutralisation des interdits théologiques et juridiques notés précédemment et la constitution de la doctrine des contrats aléatoires. De ce point de vue, il a manqué à Cournot de lire les analyses de Coumet!

Pourtant, Cournot ne nie pas que le contexte culturel dans lequel s'inscrit une découverte scientifique pèse sur le développement scientifique, puisque, on l'a vu, il insiste sur la nouvelle conception du rôle des mathématiques au XVII ${ }^{\mathrm{e}}$ siècle.

On constate donc finalement la présence, dans la pensée de Cournot, d'une tension entre l'orientation internaliste de sa représentation du développement des mathématiques et le contenu de sa philosophie de l'histoire. Cette tension témoigne, nous semble-t-il, de la difficulté, propre à l'histoire des sciences, et plus encore à l'histoire des mathématiques, de penser à la fois la nécessité interne avec laquelle s'imposent les produits de la raison et leur dépendance par rapport aux conditions historiques de leur production.

Cette difficulté trouve un écho particulièrement net dans un texte curieux de l'Essai sur les fondements de nos connaissances où Cournot s'efforce de porter un jugement sur le probabilisme antique, texte remarquable dans la mesure où Cournot y présente simultanément une thèse et le degré (faible en l'occurrence) de croyance qu'il lui attribue.

Constatant que les écrits de la Troisième Académie ne nous sont guère connus que par l'intermédiaire de Cicéron, et que l'on peut douter, malgré la lucidité dont il fait preuve, de la fidélité de sa restitution, Cournot, osant une histoire hypothétique, conjecture que ce probabilisme ait pu témoigner, grâce au secours des mathématiques, de plus de rigueur que ne lui en prête Cicéron :

«Un Grec, dont l'étude de la géométrie, alors si florissante, aurait fortifié le jugement, comme elle avait fortifié celui de Platon, et qui se nommait Arcésilas ou Carnéade, était en mesure, à ce qu'il semble, de donner à la théorie de l'opinion et de la probabilité philosophique une forme plus arrêtée que celle que nous lui trouvons dans les écrits de Cicéron et des autres anciens ${ }^{21}$. »

Mais, c'est pour aussitôt reconnaître le caractère téméraire de cette hypothèse, au regard des difficultés qu'a effectivement rencontrées et suscitées le calcul des probabilités à l'époque moderne, et la corriger :

21. Cournot, 1851, § 379; 1975, p. 448. 
«Toutefois, lorsque l'on songe que la doctrine des probabilités mathématiques est d'origine si moderne, et qu'il y a eu tant de méprises de la part des philosophes et des géomètres les plus habiles, sur la manière de l'entendre et de l'appliquer, on est bien tenté de croire que les Grecs, à leur plus belle époque, n'ont point eu à ce sujet d'idées vraiment arrêtées, et n'ont pu exprimer avec précision ce dont ils ne s'étaient pas rendu eux-mêmes un compte exact ${ }^{22}$. »

Formant l'hypothèse d'une possible mathématisation du probable dès l'Antiquité, tout en signalant simultanément son peu de vraisemblance, Cournot vient, en quelque sorte, confirmer l'analyse précédente de son aveuglement : lorsqu'il ne considère, dans les débuts du calcul des probabilités, que la technique mathématique, Cournot, constatant son caractère élémentaire, incline à penser qu'il pouvait débuter longtemps avant le $\mathrm{XVII}^{\mathrm{e}}$ siècle. Mais, dès qu'il prête attention à l'interprétation du calcul et à son application au réel, il est contraint de reconnaître que l'Antiquité grecque ne pouvait se proposer une telle entreprise. Si l'on s'en tenait à ce texte, c'est alors le point de vue internaliste de Cournot qui se trouverait ébranlé, celui-ci devant admettre que, malgré les apparences, l'Antiquité grecque ne pouvait s'engager sur la voie d'une mathématisation du hasard. Confirmation en est donnée par un autre texte extrait de l'Essai, où Cournot concède que « la notion de la probabilité n'a jamais été pour les anciens que vague et confuse », ajoutant qu'il fallut attendre «les progrès des sciences exactes » pour que naisse la théorie de la probabilité mathématique $^{23}$.

On peut trouver confirmation de l'analyse précédente dans la façon dont Cournot appréhende les premiers développements du calcul des probabilités, laquelle accuse cette distance entre l'orientation de pensée qui anime une mathématique du hasard et celle de la mathématique grecque, tout comme elle témoigne du fait qu'il considère moins l'histoire du calcul des probabilités en historien, qu'en philosophe, et en philosophe préoccupé essentiellement de la valeur objective de nos connaissances rationnelles.

Les quelques pages que consacre Cournot aux débuts du calcul des probabilités dans les Considérations mettent l'accent sur son rapport essentiel au calcul infinitésimal, soulignant par là, à la fois, que l'élément déterminant de la révolution des mathématiques au $\mathrm{XVII}^{\mathrm{e}}$ siècle est, à ses yeux, la promotion du concept d'infini comme objet mathématique, et que ce carac-

22. Cournot, 1851, § 379; 1975, p. 448-449.

23. Cournot, 1851, § 87; 1975, p. 104. 
tère révolutionnaire tient moins aux résultats que produisent les premiers probabilistes qu'aux développements ultérieurs que leurs recherches rendent possibles : le calcul des probabilités est cette « voie dont la découverte, sinon l'aplanissement, appartient au $\mathrm{XVII}^{\mathrm{e}}$ siècle » et qui, à côté de la mécanique rationnelle, constitue le second axe grâce auquel « les mathématiques nous font pénétrer dans l'économie du monde ${ }^{24}$.

De plus, Cournot, principalement préoccupé comme philosophe des probabilités de distinguer soigneusement les significations objective et subjective de la probabilité, la première permettant de prononcer des «jugements de possibilité », la seconde n'autorisant que des «jugements de simple probabilité », utilise cette distinction comme grille de lecture de l'histoire du calcul des probabilités pour constater que ses débuts sont dominés par une orientation subjectiviste :

«On voit par un passage remarquable de Pascal, qu'il ne songeait nullement aux applications que sa géométrie du hasard comportait, dans l'ordre des jugements de possibilité, et relativement à l'économie des faits naturels. Les grands génies du dix-septième siècle, Fermat, Leibnitz, Huygens, qui s'occupaient du calcul des combinaisons et des chances en même temps que Pascal ou quelques années après lui, n'avaient non plus en vue que la règle des partis ${ }^{25}$. »

Il faut attendre Jacques Bernoulli, précise Cournot, pour que la «valeur objective de la théorie des hasards » devienne le «but essentiel » du calcul. Et l'on doit alors reconnaître que la perspective à la fois subjectiviste et juridique qui préside aux premières recherches est fort éloignée de cette géométrie de la règle et du compas que pratiquaient les Grecs de l'Antiquité.

Nous avons vu que la pensée de Cournot, lorsqu'elle affronte la question des origines du calcul des probabilités, éprouve des difficultés à adopter une position claire et tranchée. Mais, pouvait-il en être autrement? Ce n'est pas seulement que notre connaissance du XvII ${ }^{\mathrm{e}}$ siècle était moins avancée en 1870 qu'aujourd'hui, c'est que le sujet est par lui-même délicat, puisqu'il s'agit de rendre compte de l'émergence de ce domaine en quelque sorte improbable et au «titre étonnant ${ }^{26}{ }^{2}$ qu'est la géométrie du hasard.

La naissance du calcul des probabilités manifeste particulièrement cette difficulté à laquelle se trouve confronté l'historien des mathématiques d'avoir à penser à la fois et conjointement la nécessité interne avec laquelle

24. Counnot, 1872, liv. III, chap. I; 1973, p. 182.

25. Cournot, $1843, \S 47 ; 1984$, p. 62.

26. Pascal, $1654 ; 1970$, p. 1035. 
se donnent les produits de la raison et les circonstances particulières de leur inscription historique; difficulté qu'entend affronter cette tradition constitutive de l'épistémologie française dont Cournot, justement, est l'un des initiateurs, et qui s'efforce de convoquer simultanément histoire et philosophie des sciences ${ }^{27}$.

Une histoire des sciences que n'éclaire pas la dimension philosophique risque fort de s'abîmer dans une érudition anecdotique où le foisonnement des détails échoue à en dissimuler la vacuité; une philosophie des sciences, oublieuse des conditions historiques d'élaboration des pratiques et des concepts qu'elle étudie, est constamment menacée de verser dans un dogmatisme projetant sur les sciences une représentation illusoire de ce qu'il voudrait qu'elles soient.

Cournot insiste sur la solidarité qui unit étroitement dans leurs développements les sciences et la philosophie ${ }^{28}$, solidarité qui, semble-t-il, dénonce l'hypothèse d'une fortuité de l'origine du calcul des probabilités. Mais il n'y a pas ici incohérence de la part de Cournot. En effet, cette solidarité n'implique pas symétrie. Il y insiste à plusieurs reprises : si les connaissances et les méthodes scientifiques nourrissent et suscitent la réflexion philosophique, seule à même d'interroger la légitimité de leurs principes et la portée de leurs résultats, la recherche scientifique n'a pas pour moteur les questions que la philosophie soulève, mais les problèmes que la science construit de l'intérieur de son propre développement. C'est pourquoi les révolutions scientifiques ne peuvent avoir, selon Cournot, qu'une origine interne ${ }^{29}$. «Si la philosophie, écrit Cournot, règne sur les sciences [...], elle ne les gouverne pas $^{30} . »$ En effet,

«l'union intime et pourtant la primitive indépendance de l'élément philosophique et de l'élément positif ou proprement scientifique dans le système de la connaissance humaine, se manifestent par ce fait bien remarquable, que l'esprit ne peut régulièrement procéder à la construction scientifique sans

27. Sans prétendre se faire ici l'interprète fidèle de la pensée d'Ernest Coumet, on peut remarquer que ce n'est pas un hasard si son séminaire du centre Alexandre-Koyré s'intitulait «Histoire et théorie » (pas plus que n'est un hasard la reprise de cet intitulé dans le titre du présent article).

28. Cournot, $1847, \S 143 ; 1989$, p. 360-363.

29. Ainsi, à propos des hypothèses de Nicolas de Cus, voir Cournot, 1872, liv. II, chap. I, p. 99-100: «Si justes que ces idées fussent, elles n'auraient pas suffi pour opérer une révolution dans la science : elles ne pouvaient servir qu'à confirmer une révolution amenée par un progrès scientifique [... car] il ne faut pas se figurer que la grande difficulté pour les partisans de l'hypothèse du mouvement de la terre fût de vaincre les préjugés des sens, des préjugés populaires, ni même de triompher de scrupules religieux : le principal obstacle venait de la science même et des données scientifiques. »

30. Cournot, $1864,1^{\text {re }}$ part., chap. VII ; 1977, p. 70. 
adopter une théorie philosophique quelconque, et que néanmoins les progrès et la certitude de la science ne dépendent point de la solution donnée à la question philosophique ${ }^{31} »$.

Thierry MARTIN

(février 2000).

31. Cournot, 1851, § 329; 1975, p. 387-388 (souligné par nous). 


\section{LISTE DES RÉFÉRENCES}

CoumEt (Ernest), 1970, «La théorie du hasard est-elle née par hasard? », Annales. Économies, sociétés, civilisations, 3, mai-juin, p. 574-598.

Cournot (Antoine-Augustin), 1973-, Eeuvres, complètes [ $=$ OC], éd. André RoBINET, Paris, Vrin (Bibliothèque des textes philosophiques).

Cournot (A.-A.), 1843, Exposition de la théorie des chances et des probabilités, Paris, Hachette, ici in $O C$, t. I, 1984.

Cournot (A.-A.), 1847, De l'origine et des limites de la correspondance entre l'algèbre et la géométrie, Paris, Hachette, ici in $O C, \mathrm{t} . \mathrm{VI} / 2,1989$.

Cournot (A.-A.), 1851, Essai sur les fondements de nos connaissances et sur les caractères de la critique philosophique, Paris, Hachette, ici in OC, t. II, 1975.

Cournot (A.-A.), 1861, Traité de l'enchaînement des idées fondamentales dans les sciences et dans l'histoire, Paris, Hachette, ici in OC, t. III, 1982.

Cournot (A.-A.), 1864, Des institutions d'instruction publique en France, Paris, Hachette, ici in $O C$, t. VII, 1977.

Cournot (A.-A.), 1872, Considérations sur la marche des idées et des événements dans les temps modernes, Paris, Hachette, ici in OC, t. IV, 1973.

Cournot (A.-A.), 1875, Matérialisme, vitalisme, rationalisme, Paris, Hachette, ici in OC, t. V, 1979.

Du Pasquier (Louis-Gustave), 1926, Le Calcul des probabilités, son évolution mathématique et philosophique, Paris, Hermann.

Gouraud (Charles), 1848, Histoire du calcul des probabilités, Paris, Auguste Durand.

LeIBNIz (Gottfried Wilhelm), 1886, Nouveaux essais sur l'entendement humain, éd. Paul JANET, Paris, Alcan.

PASCAL (Blaise), 1657, « À l'illustre académie parisienne de mathématiques », ici in Euvres complètes, éd. Jean Mesnard, Paris, Desclée de Brouwer, t. II, 1970, p. 1031-1035. 